\title{
Toward High Performance in Industrial Refrigeration Systems
}

\author{
Claus Thybo*, Roozbeh Izadi-Zamanabadi**, Henrik Niemann ${ }^{* * *}$ \\ ${ }^{*}$ Central R\&D - Refrigeration and Air Conditioning, Danfoss A/S, \\ Nordborg, Denmark, E-mail: thybo@danfoss.com \\ ** Aalborg University, Department of Control Engineering, \\ Aalborg, Denmark, E-mail: riz@control .auc.dk \\ ${ }^{* * *}$ Automation Ørsted-DTU, Technical University of Denmark, Lyngby, \\ Denmark, E-mail: hhn@oersted.dtu.dk
}

\begin{abstract}
Achieving high performance in complex industrial systems requires information manipulation at different system levels. The paper shows how different models of same subsystems, but using different quality of information/data, are used for fault diagnosis as well as robust control design in industrial refrigeration systems.
\end{abstract}

\section{Introduction}

Obtaining optimal performance in industrial refrigeration systems is the main objective in order to achieve the ultimate goals: lowering the production and maintenance costs while delivering higher level of comfort to customers. The typical characteristics of such refrigeration systems are 1-Complex nonlinear dynamics due to the refrigerants phase-shif 2- Existence of cross-coupling between different subsystems 3- High amount of disturbances imposed on the system due to an extensive interaction between the system and the environment. These characteristics makes the design of a dedicated controller a challenging task.

Optimal performance, in general, also includes the notions of high system reliability and availability. A reliable system has a certain degree of fault-tolerance. Malfunctions and faults on refrigeration systems are most often not discovered before the cooling capacity is reduced to an extent, where temperatures can no longer be maintained. In some cases no action is taken before severe damage on components or frozen goods is detected. Until action is taken the refrigeration system often runs with poor efficiency, and in some cases with higher wear on components. There are several factors that make it reasonable to include fault diagnosis functionality in the overall control system. The most important factors are: economy, safety, and the company image. In order to achieve optimal performance, different methods/approches are needed depending on the quality of information and the considered system level. On the lowest level, where it is possible to perform detailed (quantitative) modelling, complex control theory methods can be used. On the other hand, when the objective is to perform fault diagnosis on the system level, where there is a number of unknown disturbances are involved, building a detailed model is an cumbersome (almost impossible) task. Hence, qualitative based methods may be used.

The current project includes all the aspects needed for achieving optimal performance from the lowest level of the operation (controlling a valve) to the supermarket and regional level. Since this paper can not contain all these aspects, it is chosen to focus the attention on control and fault diagnosis, and illustrate how to use different approaches on the system in order to obtain different objectives.

\section{Refrigeration system}

The refrigeration system illustrated in Figure 1 shows the operation of the system. The refrigeration display cabinet, illustrated in Figure 1, operates by applying a duvet of cold air over the food to keep the food cold.

A fan circulates the air through the evaporator, over the load zone with the food, and down to the inlet of the evaporator. During the circulation, a fraction of the cooled air is lost in the load zone and replaced with ambient air. Radiation penetrating the cold duvet presents a sensitive load that heats up the air in the load zone.

\subsection{Evaporator model}

The function of the evaporator is to absorb the heat from the passing air to compensate for the sensitive load and air mixing in the cabinet. The evaporation system is divided into two interacting processes; the one involves the refrigerant and the other involves the air.

Mathematical models that include the dynamic behavior of refrigerant are complex and contain uncertainties due to the complexity of the refrigerant boiling process [7]. The type of the considered faults and their impact on the system does not necessitates the use of dynamic equations for the boiling of the refrigerant. Hence, only a steady state model of the system is considered for fault diagnosis. 


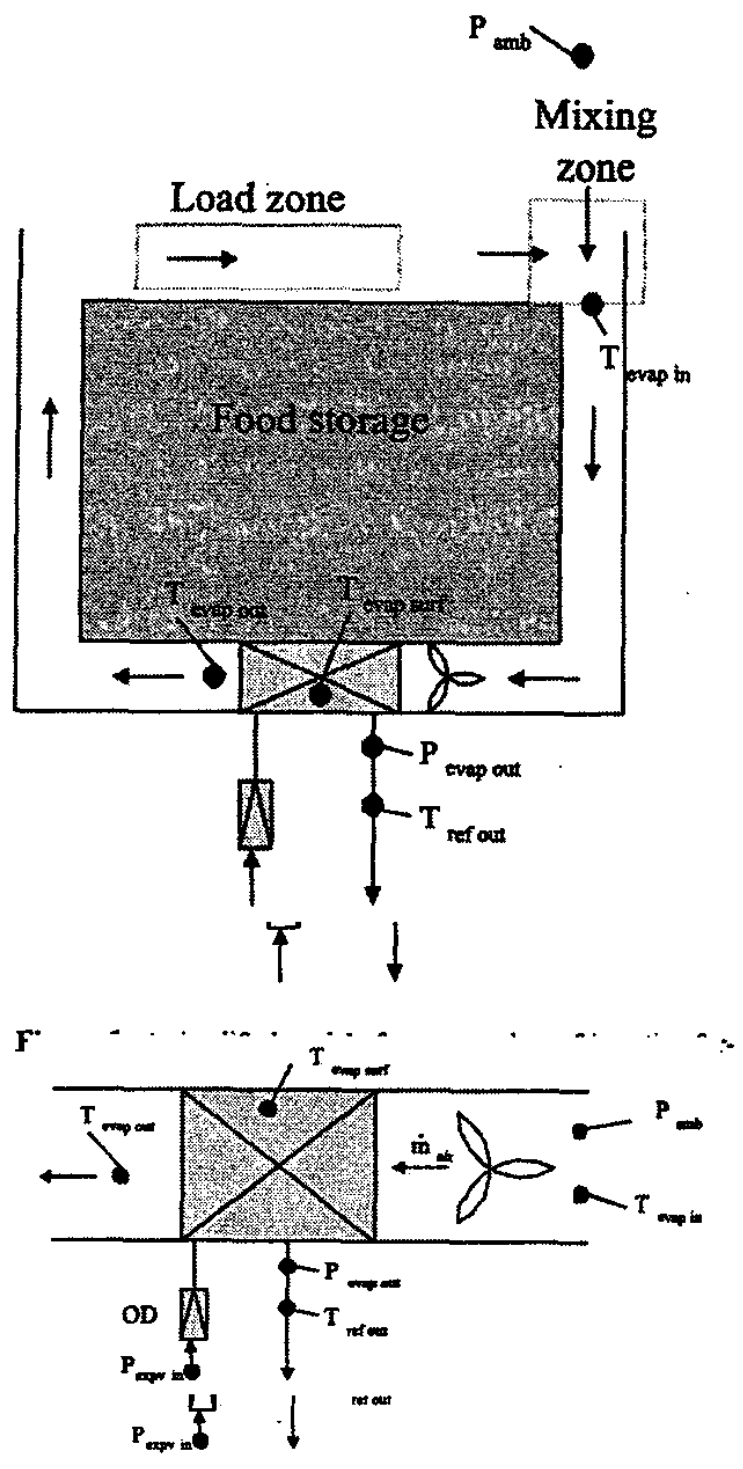

Figure 2: A simplified model of an air cooled evaporator.

2.1.1 The refrigerant side of the evaporator: The relations governing the refrigerant side of the evaporator are:

$$
\begin{array}{ll}
f_{1}: & \dot{Q}_{E}=\dot{m}_{r e f}\left(h_{r e f, \text { out }}-h_{r e f, \text { in }}\right) \\
f_{2}: & h_{\text {ref,in }}=g_{1}\left(T_{\text {ref,in }}\right) \\
f_{3}: & h_{\text {ref,out }}=g_{2}\left(T_{\text {ref,out }}, P_{\text {evap }, \text { out }}\right) \\
f_{4}: & \dot{m}_{\text {ref } f}=g_{3}\left(O D, P_{C}, P_{\text {eva }, \text { out }}, T_{\text {expv,in }}\right) .
\end{array}
$$

$\dot{Q}_{E}$ represents the obtained heat by the refrigerant. $h_{\text {ref }, \text { in }}$ $\left(h_{r e f, o u n}\right)$ are the refrigerant's enthalpy at the input (output) of the evaporator and $\dot{m}_{\text {ref }}$ is the mass flow of the refrigerant through the evaporator. $g_{1}$ and $g_{2}$ represent tables of data. $g_{3}$ is also a table of data representing the behavior of the expansion valve at different operating points. The various measurements of temperature and pressure are shown in figure 2. $O D$, which is the opening degree of the expansion valve, is the control signal to the evaporation system. $f_{i}, \quad(i=1, \ldots, 4)$ represent relations/constraints goveming the involved variables. For instance, the relation $f_{1}$ governs the variables $\dot{Q}_{E}, \dot{m}_{r e f}, h_{\text {ref,out }}$, and $h_{r e f, i n}$, hence $f_{1}\left(\dot{Q}_{E}, \dot{m}_{\text {ref }}, h_{\text {ref,out }}, h_{\text {ref, in }}\right)=0$. These relations are used in sections 3 and 4 to perform the structural analysis of the system.

2.1.2 The air side of the evaporator: Air passing the evaporator is assumed to have a uniform inlet and outlet temperature and relative humidity $(R H)$. The involved variables are: air temperature $T_{\text {air, } i n}, T_{\text {air,our }}$, the partial pressure of the water vapor (in the air) $P_{d, i n}, P_{d, \text { out }}$, air's ambient pressure $P_{a m b}$, humidity ratio $x_{i n}$ and $x_{\text {out }}$, air enthalpy $h_{a i r, i n}$ and $h_{a i r, o u t}$ and the transferred heat $\dot{Q}_{a i r}$. The governing relations are:

$$
\begin{aligned}
& f_{5}: \dot{Q}_{a i r}=\dot{m}_{a i r}\left(h_{\text {air, in }}-h_{\text {air }, \text { out }}\right) \\
& f_{6}: h_{\text {air, in }}=g_{4}\left(T_{\text {air, in }}, x_{\text {air,in }}\right) \\
& f_{7}: h_{\text {air,out }}=g_{4}\left(T_{\text {air,out }}, x_{\text {air,out }}\right) \\
& f_{8}: x_{a i r, i n}=0.622 * P_{d, \text { in }} /\left(P_{a m b}-P_{d, i n}\right) \\
& f_{9}: x_{\text {air,out }}=0.622 * P_{d, \text { out }} /\left(P_{\text {amb }}-P_{d, \text { out }}\right) \\
& f_{10}: \underline{P}_{d, \text { in }}=g_{5}\left(R H, T_{\text {air, in }}\right) \\
& f_{11}: \quad P_{d, \text { out }}=g_{5}\left(R H, T_{\text {air,out }}\right) \text {. }
\end{aligned}
$$

$-g_{4}$ and $g_{5}$ represent tables of experimentally obtained data. The air mass flow $\dot{m}_{\text {air }}$ is a variable which has a constant mean value during the normal operation.

\section{Structural modeling}

Consider the system $S$ as a set of components $\bigcup_{i=1}^{m} C_{i}$, each imposing one (or several) relations $f_{i}$ between a set of variables $z_{j}, j=1, \ldots, n$ i.e.

$$
f_{i}\left(z_{1}, \ldots, z_{p}\right)=0, \quad 1<p \leq n
$$

$f_{i}$ can represent any kind of relation (dynamic, static, linear, or non-linear). (These relations are also called constraints as the value of an involved variable can not change independently of the other involved variables ([2], [3]). The system's structural model is represented by the set of relations $\mathscr{F}=\left\{f_{1}, f_{2}, \cdots, f_{m}\right\}$ and the set of variables $\mathscr{Z}=$ $\mathscr{X} \cup \mathscr{X}=\left\{z_{1}, z_{2}, \cdots, z_{n}\right\} . \mathscr{X}$ is the set of unknown variables and $\mathscr{K}=\mathscr{U} \cup \mathscr{Y}$ is the set of known variables i.e. input/reference signals $(\mathscr{U})$, and measured signals $(\mathscr{Y})$.

\subsection{Structural model representation}

The system's structural model can be represented by a bipartite graph, $G(\mathscr{F}, \mathscr{Z}, \mathscr{A})$ where elements in the set of arcs $\mathscr{A} \subset \mathscr{F} \times \mathscr{Z}$ are defined in a certain way. To specify the elements in the set $\mathscr{A}$ in a useful manner, an additional property that is the calculability property, needs to be taken 


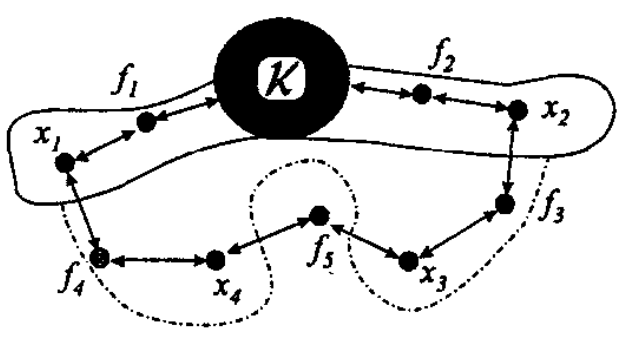

Figure 3: The process of matching.

into considerations.

Calculability property: Let $z_{j}, j=1, \cdots, p, \cdots, n$ be variables that are related through a constraint $f_{i}$, e.g. $f_{i}\left(z_{1}, \cdots, z_{n}\right)=0$. The variable $z_{p}$ is calculable if its value can be determined through the constraint $f_{i}$ under the condition that the values of the other variables $z_{j}, j=1, \cdots, n, j \neq$ $p$ are known. Taking calculability properties into considerations, the systems structural model is now represented by a bipartite directed graph: The structure graph of the system is a bipartite directed graph $(\mathscr{F}, \mathscr{Z}, \mathscr{A})$ where the elements in the set of $\mathscr{A} \subset(\mathscr{F} \times \mathscr{X})$, where $\mathscr{Z}=\mathscr{X} \cup \mathscr{X}$ are defined by:

$$
\begin{cases}a_{i j}=\left(f_{i}, x_{j}\right)=1 & \text { iff } f_{i} \text { applies to } x_{j} \\ a_{i j}^{*}=\left(x_{i}, f_{j}\right)=1 & \text { iff } x_{i} \text { is calculable through } f_{j} \\ k f_{i}=\left(k_{i}, f_{j}\right)=1 & \text { iff } f_{j} \text { applies on known var. } k_{i} \\ 0 & \text { Otherwise. }\end{cases}
$$

for all $x \in \mathscr{X}$ and $k \in \mathscr{X}$.

\subsection{Matching}

The main purpose of developing a matching algorithm is to identify the subsystem(s), which contain redundant information. The idea is depicted in figure 3 . The algorithm initiates the matching from the known variables. The figure illustrates the idea of making the unknown variable "known" by successively matching them to previously known variables. First, variables $x_{1}$ and $x_{2}$ are matched to constraints $f_{1}$ and $f_{2}$ (full line). These variables become "known" as all the other variables that enter $f_{1}$ and $f_{2}$ are known. Hence, the new set of known variables can be considered as $\mathscr{K}_{\text {new }}=\mathscr{K} \cup x_{1} \cup x_{2}$. Next, $x_{3}$ and $x_{4}$ are matched to $f_{3}$ and $f_{4}$ correspondingly (dotted line) etc. The procedure is repeated until a stop criteria is met.

\section{Fault diagnosis}

A typical fault that occurs in industrial refrigeration systems is a breakdown in one or several fans. When a fan breaks down the air circulation will be reduced or stopped. The consequence is a reduced transfer of heat from the circulating air caused by a reduced mass flow of the air, $\dot{m}_{\text {air }}$. The overheating control system takes action by closing the expansion valve. Seen from the cooling control system a fan breakdown just reduces the needed cooling effect of the system. The fan operates at constant speed in normal operation, and is assumed to stop or operate with a reduced speed in case of a fault. Hence an estimate for the mass-flow of the air can be expressed as a constant, $\dot{\bar{m}}_{a i r}$. This is represented by the following relation:

$$
f_{12}: \quad \dot{\dot{m}}_{a i r}=\dot{m}_{a i r}
$$

The final relation that relates the air side of the evaporator to the refrigerant side is:

$$
f_{13}: \quad \dot{Q}_{E}=\dot{Q}_{a i r}
$$

\subsection{Structural analysis of evaporation system}

The fault diagnosis possibility in the evaporation system is evaluated in the following:

The structural model of the system is defined by the sets: $\mathscr{F}=\left\{f_{1}, \ldots, f_{13}\right\}, \mathscr{K}=\left\{P_{C}, O D, P_{\text {evap }, \text { out }}, T_{\text {app }, \text { in }}\right.$, $\left.T_{\text {ref,out }}, T_{\text {ref,in }}, T_{a i r, \text { oun }}, R H, T_{a i r, i n}, P_{a m b}\right\}$, and $\mathscr{X}=\left\{\dot{m}_{\text {ref }}\right.$, $h_{r e f, \text { out }}, h_{r e f, i n}, \dot{Q}_{E}, \dot{Q}_{a i r}, \dot{m}_{\text {air }}, h_{\text {air,out }}, h_{\text {air }, \text { in }}, x_{\text {air,out }}$, $\left.x_{a i r, i n}, P_{d, \text { ous }}, P_{d, i n}\right\}$.

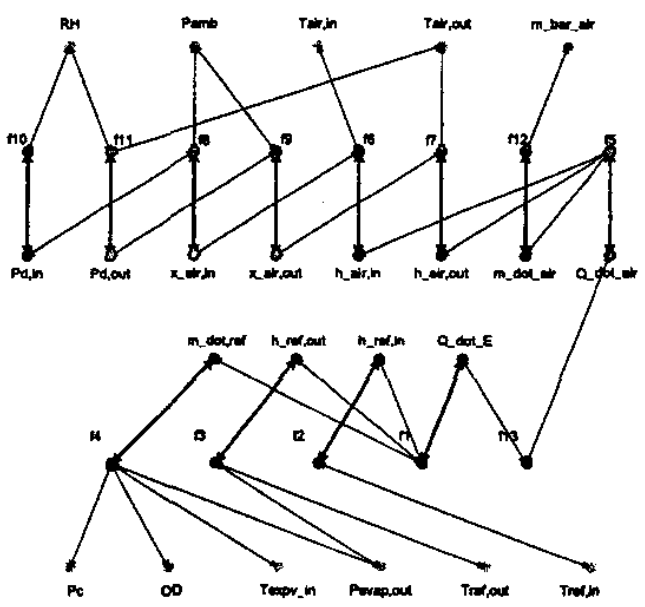

Figure 4: The digraph model representation of the evaporation system.

Figure 4 illustrates the resulting structural model of the evaporation system, represented by a digraph. The matching result is illustrated by thick arrows on the figure. It is shown that there is one unmatched relation, i.e. $f_{13}$, which can be used for fault detection purposes. The unmatched relation represents one analytical redundancy in the evaporation system. According to relation $f_{13}$

$$
f_{13}\left(\dot{Q}_{E}, \dot{Q}_{a i r}\right)=0 \quad: \quad \dot{Q}_{E}-\dot{Q}_{a i r}=0
$$

The equality should hold during normal operation. The matching shows that the values of the variables $\dot{Q}_{E}$ and $\dot{Q}_{a i r}$ can be computed, through the involved relations, from their 
related known variables, i.e. there exists functions $g_{Q_{e}}$ and $g_{\text {Qair }}$ such that

$$
\begin{aligned}
\dot{Q}_{E} & =g_{Q e}\left(P_{c}, O D, T_{\text {expv,in }}, P_{\text {evap,out }}, T_{\text {ref,out }}, T_{\text {ref, in }}\right) \\
\dot{Q}_{\text {air }} & =g_{\text {Qair }}\left(R H, P_{\text {amb }}, T_{a i r, i n}, T_{\text {air,out }}\right) .
\end{aligned}
$$

It should be noticed that the notion of time in the equations is omitted for brevity reasons. It is possible to define a residual,

$$
r(t)=\dot{Q}_{E}(t)-\dot{Q}_{a i r}(t)
$$

which will have an expected mean value equal to zero in normal operation and will deviate from zero under faulty conditions.

\section{Feedback Control of the refrigeration system}

The control strategy for the refrigeration system is described in the following. First, an analysis of the system is given followed by a setup for the design of a feedback controller.

\subsection{System Analysis}

The static non-linear model described in Section 2 cannot be applied in connection with design of dynamic feedback controllers. Instead, dynamic models of the refrigeration system need to be derived. This has been done in [4]. Both a full nonlinear model, a SIMULINK model of the refrigeration system and a number of linear models in some working points has been described in [4], based on the real lab system.

Linearizing the nonlinear model, we can describe the system by the following MIMO state space model:

$$
\begin{aligned}
& \dot{x}=A x+B_{1} d+B_{2} u \\
& y=C x+D d
\end{aligned}
$$

where $x$ is the state vector, $u$ is the control input vector, $d$ is the disturbance on the system and $y$ is the measurement vector. All the state matrices in (19) depend on the working point. The model consist of 11 states, 6 states for the model of the condenser and 5 states for the model of the evaporator. The control inputs to the system are the speed of the pump and the opening of the valve. The measurement outputs from the system are temperatures at the overheating. Changes in outside temperatures at the condenser and at the evaporator side comprises the system's disturbance signals.

The four open loop transfer functions are shown in Figure 5 for the nominal working point. The inputs and outputs have been scaled such that the variables are in the interval $[-1,1]$.

From Figure 5, it is quite clear that the system need to be considered as a MYMO system, due to the cross coupling terms. The linear model will depend on the working

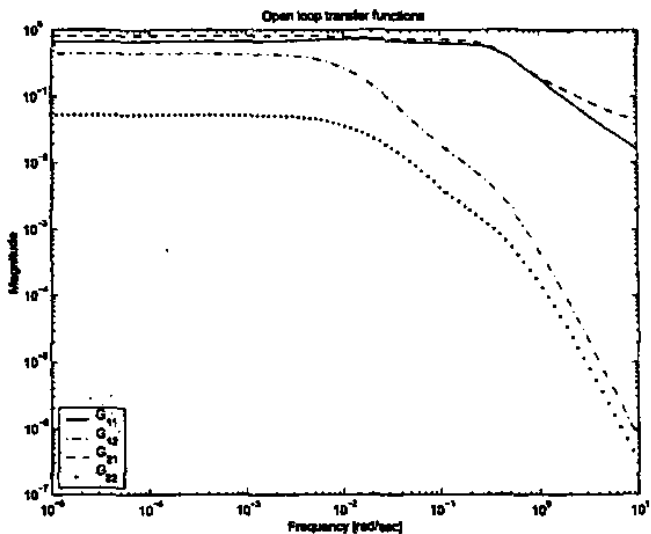

Figure 5: The four open loop transfer functions of the refrigeration system.

point. Linearizing the nonlinear model around other working points that the nominal working point, the main difference is a change in the amplitudes of the transfer functions.

\subsection{Control Strategy}

The design of a feedback controller for the system is based on the standard setup for robust controller design, described in e.g. [5]. The standard setup is described by:

$$
\left(\begin{array}{l}
z \\
y
\end{array}\right)=P\left(\begin{array}{l}
w \\
u
\end{array}\right)
$$

where $P$ is the generalized plant, $w$ is an extemal input, $z$ is the external output to be controlled, i.e. minimize the effect from $w$ on $z, y$ and $u$ are the measurement and the control signal, respectively.

In the design of a feedback controller for the refrigeration system, we want to minimize the effect from external disturbance, i.e. changes in the external temperature. Furthermore, an estimate of the heat transfer rate $\dot{Q}_{E}$ is needed in connection with the feedback controller. The estimate of $\dot{Q}_{E}$ need to be applied in connection with the fault diagnosis of the system, see Section 4. When the system include uncertainties (including non-linearity), the design of the feedback controller and the estimator need to be a combined design, see e.g. [6].

The estimation of $\dot{Q}_{E}$ consists of two parts:

a) An estimation of the mass flow, $\dot{m}_{r e f}$, which is a function of the pressure difference over the expansion valve given by:

$$
\begin{aligned}
\hat{m}_{r e f} & =\alpha_{o p e n} \beta \sqrt{\left(\hat{P}_{\text {exp }, o u t}-\hat{P}_{\text {expv,in }}\right)} \\
& =\alpha_{o p e n} \beta \sqrt{\Delta \bar{P}_{\text {expv }}}
\end{aligned}
$$

where $\alpha_{o p e n}$ is the opening of the valve and $\beta$ is a constant parameter.

b) An estimation of the difference between the refrigerant's enthalpy at the output and the input, $h_{\text {ref,out }}-h_{\text {ref, in }}=$ 
$\Delta h_{r e f}$. The estimation of $\Delta P_{\text {exp }}$ and $\Delta h_{r e f}$ can easily be included in the standard setup. Let the control vector be given by

$$
u=\left(\frac{u_{1}}{u_{2}}\right)=\left(\begin{array}{c}
\frac{u_{\text {feedbact }}}{\Delta \hat{P}_{\text {expv }}} \\
\Delta \hat{h}_{\text {ref }}
\end{array}\right)=K y
$$

The external output vector $z$ is extended by the estimation error of $\Delta P_{\text {expv }}$ and $\Delta h_{\text {ref }}$, i.e.

$$
z=\left(\begin{array}{c}
z_{1} \\
e_{\Delta P} \\
e_{\Delta h}
\end{array}\right)=\left(\begin{array}{c}
z_{\text {feedback performance }} \\
\Delta \dot{P}_{\text {exp }}-\Delta \tilde{P}_{\text {expv }} \\
\Delta h_{r e f}-\Delta \hat{h}_{r e f}
\end{array}\right)
$$

\section{Experimental results}

The following two subsections present the obtained results for controller design as well as fault diagnosis results for the considered fan faults.

\subsection{Controller design Results}

The focus in this section will be on the design of the filter/observer $K_{\text {est }}$ for estimation of $\Delta P_{\text {expv }}$ and $\Delta h_{r e f}$, where the guidelines given in [6] were followed. It tums out that the dominant part in the design of a feedback controller/estimator is the design of the feedback controller. One reason is that the system include a non-minimum phase zero close to the imaginary axis, which limited the performance of the closed loop system.

The design of the estimator is based on a minimization of the estimation errors at low frequencies. This is obtained by using a lowpass weight function on the estimation error given by (23). The results of the design of an estimator is shown in Figure 6 - 8.

The four transfer functions of the estimator is shown in Figure 6. It can be seen that the estimator is constant at low frequencies. An approximation of the high order estimator with first order low pass filters can be done without reducing the performance of the estimation of $\Delta P_{\text {exp }}$ and $\Delta h_{\text {ref }}$. This result is also in line with the open loop transfer function of the system shown in Figure 5.

The transfer function from the external input $T_{\text {exap }}$ to the estimation errors is shown in Figure 7. As it can be seen, the estimation errors at low frequencies are very small. In the design of the estimator, this error can further be minimized. A time response of the estimation errors is shown in Figure 8. The result is in line with Figure 7, the estimation errors are quite small, the errors approach zero very fast, around 5 - 10 times faster that the closed-loop dynamic. The reason is the almost static behaviour at low frequencies, where the system is operating.

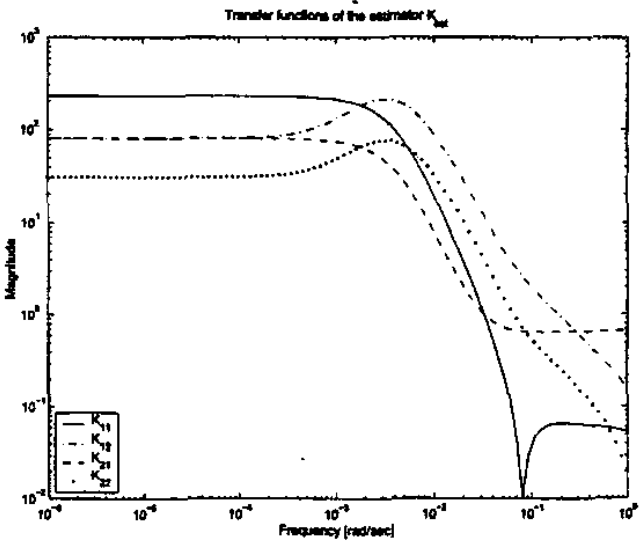

Figure 6: The transfer functions of $K_{\text {est }}$.

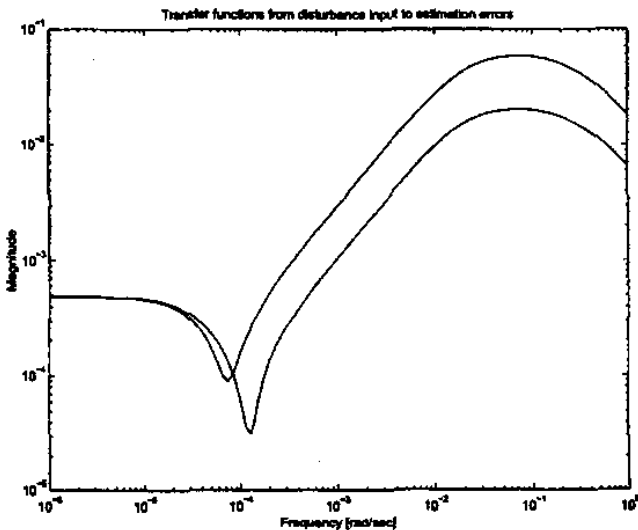

Figure 7: The transfers function from the extemal disturbance input $T_{\text {evap }}$ to the estimation errors, $e_{\Delta P}$ and $e_{\Delta t}$.

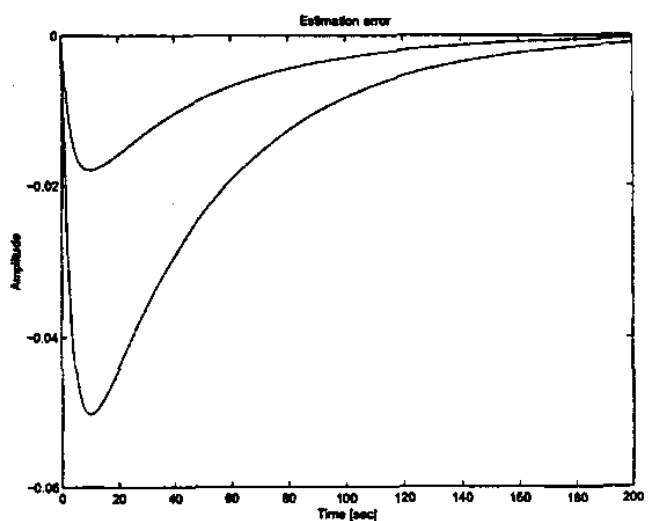

Figure 8: The estimation error responses for a step at the external input $T_{\text {evap. }}$.

\subsection{Fault diagnosis results}

The following fault detection results have been obtained by performing experiments on an refrigerated island cabinet 
display dedicated for experiments. The experimental result in Figure 9 shows the residual of a scenario where two single fan faults were followed by a double fan fault. The un-

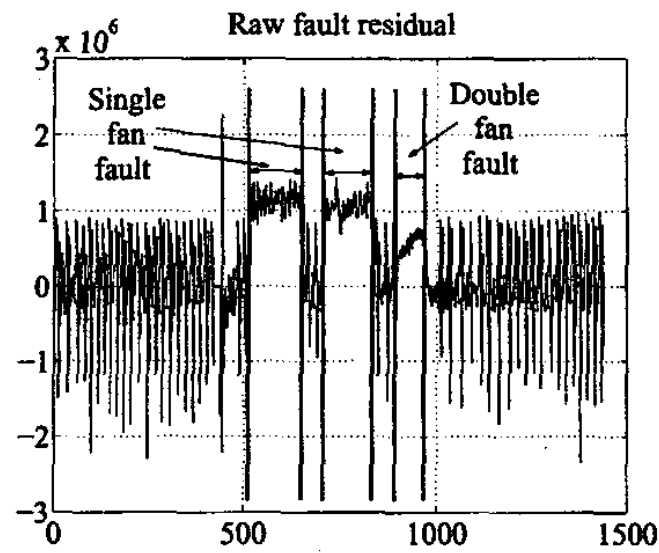

Figure 9: The normalized unfiltered residual during a fan-fault scenario. The time-axis is in minutes.

filtered residual in Figure 9contains a strong noise component, but aiso a significant sensitivity to the faults. The high frequency noise component is explained by the steady state model that do not embrace the higher order dynamics of the system. This noise can be approximated by a white noise (in no fault situations) with zero mean value and a proper variance. The faulty situation can then be detected by an

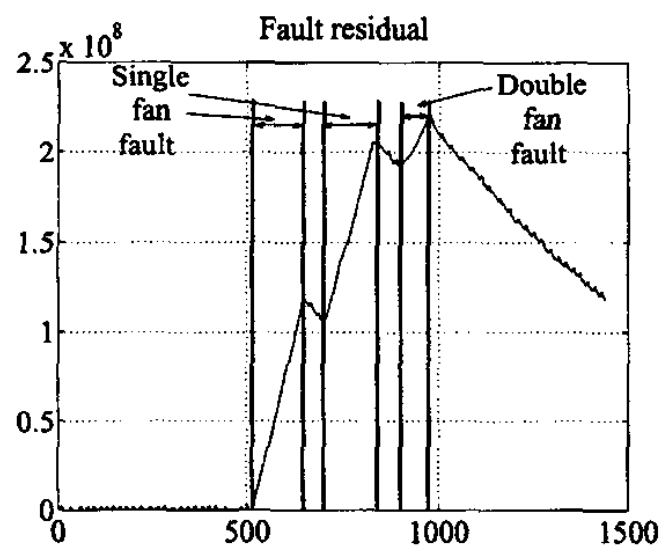

Figure 10: The normalized cusum filtered residual during a fanfault scenario. The time-axis is in minutes.

appropriate statistical based algorithm such as the CUSUM algorithm [1] as illustrated in Figure 10. An acceptable time to detect for a single fan fault is 60 minutes, which easily can be met in this experiment.

\section{Concluding remarks}

The objectives of this paper was to illustrate how different modelling approaches for the same subsystems can be used to achieve different goals in industrial systems. In order to obtain optimal control performance and reliability in the evaporation system of industrial control systems two different approaches where used to build a qualitative and a quantitative model of the subsystem. The qualitative model was used to obtain information about the monitoring possibility in the system and furthermore, to obtain redundant information that could be used for fault diagnosis purpose. The quantitative(detailed) model was used to analyze and then design a robust controller for the considered subsystem.

\section{Acknowledgement}

The authors would like to acknowledge the financial and technical expertise that was obtained through cooperation within the EFP project (ESO).

\section{References}

[1] M. Basseville and I. V. Nikiforov. Detection of Abrupt Changes: Theory and Application. Information and System Science. Prentice Hall, New York, 1993.

[2] J. Ph. Cassar, R. G. Litwak, V. Cocquempot, and M. Staroswiecki. Approche structurelle de la conception de systèmes de surveillance pour des procédé industriels complexes. Revue Européenne de Diagnostic et Sûreté de fonctionnement, 1994. Vol.4, no. 2, pp. 179-202.

[3] P. Declerck and M. Staroswiecki. Characterization of the canonical components of a structural graph for fault detection in large scale industrial plants. In Proceedings of ECC'91, pages 298-303, Grenoble, France, July 1991.

[4] L. Larsen and J. Holm. Model of a refrigeration system. Preliminary results from a master project at Technical University of Denmark.

[5] S. Skogestad and I. Postlethwaite. Multivariable feedback control: Analysis and Design. Wiley, 1996.

[6] J. Stoustrup, M.J. Grimble, and H.H. Niemann. Design of integrated systems for control and detection of actuator/sensor faults. Sensor Review, 17:157-168, 1997.

[7] W.-D.Gruhle and R. Isermann. Modelling and control of a refigerant evaporator. pages 287-292, Boston, USA, 1985. American Control Conference. 